OPEN ACCESS

Edited by:

David Hains,

Riley Hospital for Children,

United States

Reviewed by:

Reinhard Berner,

Technische Universität

Dresden, Germany

Yuhan Xing,

The Chinese University of Hong

Kong, China

*Correspondence:

Silvia Garazzino

silvia.garazzino@unito.it

Specialty section:

This article was submitted to

Pediatric Infectious Diseases,

a section of the journal

Frontiers in Pediatrics

Received: 04 January 2021 Accepted: 15 February 2021

Published: 16 March 2021

Citation:

Garazzino S, Lo Vecchio A, Pierantoni L, Calò Carducci Fl, Marchetti F, Meini

$A$, Castagnola E, Vergine $G$, Donà $D$,

Bosis S, Dodi I, Venturini E, Felici E,

Giacchero R, Denina M, Pierri L,

Nicolini G, Montagnani C, Krzysztofiak

A, Bianchini S, Marabotto C, Tovo

P-A, Pruccoli G, Lanari M, Villani A

Castelli Gattinara $G$ and the Italian SITIP-SIP Pediatric Infection Study Group (2021) Epidemiology, Clinical

Features and Prognostic Factors of

Pediatric SARS-CoV-2 Infection:

Results From an Italian Multicenter

Study. Front. Pediatr. 9:649358.

doi: 10.3389/fped.2021.649358

\section{Epidemiology, Clinical Features and Prognostic Factors of Pediatric SARS-CoV-2 Infection: Results From an Italian Multicenter Study}

Silvia Garazzino ${ }^{1 *}$, Andrea Lo Vecchio ${ }^{2}$, Luca Pierantoni ${ }^{3}$, Francesca Ippolita Calò Carducci ${ }^{4}$, Federico Marchetti ${ }^{5}$, Antonella Meini ${ }^{6}$, Elio Castagnola ${ }^{7}$, Gianluca Vergine ${ }^{8}$, Daniele Donà ${ }^{9}$, Samantha Bosis ${ }^{10}$, Icilio Dodi ${ }^{11}$, Elisabetta Venturini ${ }^{12}$, Enrico Felici ${ }^{13}$, Roberta Giacchero ${ }^{14}$, Marco Denina ${ }^{1}$, Luca Pierri ${ }^{2}$, Giangiacomo Nicolini ${ }^{15}$, Carlotta Montagnani ${ }^{12}$, Andrzej Krzysztofiak ${ }^{4}$, Sonia Bianchini ${ }^{16}$, Caterina Marabotto ${ }^{4}$, Pier-Angelo Tovo ${ }^{1}$, Giulia Pruccoli ${ }^{1}$, Marcello Lanari ${ }^{3}$, Alberto Villani ${ }^{4}$, Guido Castelli Gattinara ${ }^{4}$ and the Italian SITIPSIP Pediatric Infection Study Group

\footnotetext{
${ }^{1}$ Pediatric Infectious Diseases Unit, Regina Margherita Children's Hospital, University of Turin, Turin, Italy, ${ }^{2}$ Section of Pediatrics, Department of Translational Medical Science, University of Naples Federico II, Naples, Italy, ${ }^{3}$ Pediatric Emergency Unit, IRCCS Azienda Ospedaliero-Universitaria di Bologna, Bologna, Italy, ${ }^{4}$ Universitarian-Hospital Department, Ospedale Bambino Gesù IRCCS, Rome, Italy, ${ }^{5}$ Department of Pediatrics, Santa Maria delle Croci Hospital, Ravenna, Italy,

${ }^{6}$ Department of Experimental and Clinical Sciences, Pediatric Clinic, University of Brescia, Brescia, Italy, ${ }^{7}$ Infectious Diseases Unit, IRCCS Istituto Giannina Gaslini, Genoa, Italy, ${ }^{8}$ UOC Pediatria, Ospedale degli Infermi di Rimini, Rimini, Italy, ${ }^{9}$ Division of Pediatric Infectious Diseases, Department of Women's and Children's Health, University Hospital of Padua, Padua, Italy,

${ }^{10}$ Fondazione IRCCS Cà Granda Ospedale Maggiore Policlinico, Milan, Italy, ${ }^{11}$ Emergency and General Pediatric Unit, Pietro Barilla Children's Hospital, Parma, Italy, ${ }^{12}$ Infection Disease Unit, Meyer Children's University Hospital, Florence, Italy,

${ }^{13}$ Pediatric and Pediatric Emergency Unit, The Children Hospital, AO SS Antonio e Biagio e C. Arrigo, Alessandria, Italy, ${ }^{14}$ UOC Pediatria ASST di Lodi, Lodi, Italy, ${ }^{15}$ UOC Pediatria, San Martino Hospital, Belluno, Italy, ${ }^{16}$ Department of Pediatrics, ASST Santi Paolo e Carlo Hospital, Milan, Italy
}

Background: Many aspects of SARS-CoV-2 infection in children and adolescents remain unclear and optimal treatment is debated. The objective of our study was to investigate epidemiological, clinical and therapeutic characteristics of pediatric SARS-CoV-2 infection, focusing on risk factors for complicated and critical disease.

Methods: The present multicenter Italian study was promoted by the Italian Society of Pediatric Infectious Diseases, involving both pediatric hospitals and general pediatricians/family doctors. All subjects under 18 years of age with documented SARS-CoV-2 infection and referred to the coordinating center were enrolled from March 2020.

Results: As of 15 September 2020, 759 children were enrolled (median age 7.2 years, IQR 1.4; 12.4). Among the 688 symptomatic children, fever was the most common symptom (81.9\%). Barely $47 \%$ of children were hospitalized for COVID-19. Age was inversely related to hospital admission $(p<0.01)$ and linearly to length of stay $(p=0.014)$. One hundred forty-nine children (19.6\%) developed complications. Comorbidities were risk factors for complications $(p<0.001)$. Viral coinfections, underlying clinical conditions, age $5-9$ years and lymphopenia were statistically related to ICU admission $(p<0.05)$. 


\begin{abstract}
Conclusions: Complications of COVID-19 in children are related to comorbidities and increase with age. Viral co-infections are additional risk factors for disease progression and multisystem inflammatory syndrome temporarily related to COVID-19 (MIS-C) for ICU admission.
\end{abstract}

Keywords: SARS-CoV-2 infection, children, multisystem inflammatory syndrome, MIS-C, COVID-19

\section{INTRODUCTION}

Since December 2019 Coronavirus Disease (COVID-19) caused by severe acute respiratory syndrome coronavirus 2 (SARS$\mathrm{CoV}-2$ ) has rapidly spread, becoming the first pandemic of the 21 st century for number of deaths (1). From the available data, children appear to be less affected than adults, with a significantly lower mortality rate, although severe complications may also occur (2-6). However, many aspects of SARS-CoV-2 infection in children and adolescents remain unclear, optimal treatment is debated and the role of young children as drivers of viral transmission is under discussion.

We hereby report the results of a national multicentre study aimed at investigating epidemiological, clinical and therapeutic aspects of SARS-CoV-2 infection in infants, children and adolescents, hereafter referred to as pediatric population or children.

\section{MATERIALS AND METHODS}

The present study was promoted by the Italian Society of Pediatric Infectious Diseases, with the endorsement of the Italian Society of Pediatrics and involved 11 of 13 exclusively pediatric hospitals, 51 pediatric units across Italy as well as general pediatricians. All subjects with $<18$ years of age with documented SARS-CoV-2 infection were recruited. Diagnosis of infection was established in presence of at least one respiratory specimen positive for SARS-CoV-2 nucleic acid using a validated real-time reverse-transcriptase polymerase-chain-reaction (RTPCR) assay. All molecular tests fulfilled performance criteria established by the European Commission (7) and tested envelope protein gene (E), nucleocapsid protein gene $(\mathrm{N})$ and RNAdependent RNA polymerase gene (RdRp). Only highly suggestive symptomatic children with COVID-positive households were diagnosed through detection of IgM and IgG antibodies against SARS-CoV-2 (DiaSorin Inc.) if molecular testing was not available (as occasionally occurred in March-April due to shortage of reagents). Children were generally retested after 14 days from the first positive molecular test, and then weekly until a negative result.

Medical records of all enrolled patients were revised as of 15 September 2020. Data were de-identified, recorded through a targeted registration form and transferred to a specifically designed database. Data collection was allowed by written consent of at least one parent for active participation to the study.

The study received ethical approval on 24 March 2020 (protocol number 0031296).

An adequate follow-up to outline the outcome of the infection was required, in most instances at least 2 weeks.
Children were categorized into 3 groups according to the healthcare setting: children hospitalized for COVID-19-related reasons; children with SARS-CoV-2 infection but hospitalized for reasons other than COVID-19; outpatients, including children entirely followed by their general pediatrician or children visited and tested in the emergency department but not hospitalized.

The COVID-19-related complications were defined as follows: (a) clinical and/or radiological diagnosis of pneumonia; (b) severe acute respiratory illness ( $\mathrm{SpO} 2<92 \%$ associated with tachypnea and other signs of respiratory failure); (c) acute respiratory distress syndrome; (d) neurological disturbances; (e) severe dehydration requiring intravenous rehydration; (f) severe bacterial supra-infection; (g) specific involvement of a single organ/apparatus requiring hospitalization (i.e., myocarditis, pericarditis, pancreatitis, etc.); (h) multisystem inflammatory syndrome temporarily related to COVID-19 (MIS-C) according to CDC criteria (8).

Statistical analysis was performed using IBM SPSS Statistics 25.0 (IBM Corp. Armonk, NY). Statistical significance was set at $p<0.05$. All $p$-values were 2 -tailed. In the descriptive analysis categorical variables were expressed as percentage and continuous variables as mean, median and interquartile range (IQR). To compare continuous variables of the study groups, Student $t$-test was used. To evaluate discrete variables, Pearson $\chi^{2}$ and correlation Fisher exact tests were performed as appropriate. One-way analysis of variance (ANOVA) was used for linear regression analysis.

\section{RESULTS}

As of 15 September 2020, 759 children were enrolled (426, $56.1 \%$, males-Table 1 ). The mean age was 7.3 years (median 7.2 years, IQR: $1.4-12.4$ years). Of them, 160 (21.1\%) were infants, including 40 neonates ( 3 preterm).

The majority of children $(535 / 759,70.5 \%)$ had at least one infected parent, with the mother being carrier in $60 \%$ of cases. Close contact with other infected households was reported in $76(10.0 \%)$ subjects; 377 children $(49.7 \%)$ had more than one infected family member.

Of the 569 children with known immunization status, only 26 (4.6\%) had received seasonal 2019-2020 flu vaccine. One hundred and thirty-six children (17.9\%) had underlying chronic diseases (Table 1); forty (5.3\%) had a history of premature birth.

\section{Clinical Presentation}

Ninety-one (12.0\%) children were asymptomatic: they were screened either for a contact with a SARS-CoV-2 positive person or during hospitalization for other reasons. None developed symptoms at follow-up. 
TABLE 1 | Characteristics of enrolled children according to health-care setting.

Total $(n=759)$
Outpatients
$(n=371,48.9 \%)$
Inpatients $(n=388,51.1 \%)$

Admitted for other reasons

\section{Age (years)}

Mean, median (IQR)

$7.3,7.2(1.4-12.4)$

8.6, $9.2(3.7-13.2)$

5.8, $3.8(0.5-10.8)$

Age groups

$$
\begin{aligned}
& <1 y \\
& 1-4 y \\
& 5-9 y \\
& 10-17 y
\end{aligned}
$$

\section{Gender}

$$
\text { Males }
$$

Females

\section{Underlying chronic diseases}

Total

Congenital malformations

Asthma

Epilepsy

Complex genetic syndromes

Endocrine disorders

Cancers

Hematologic diseases

Rheumatologic diseases

Autism or neurological development impairment

Gastrointestinal diseases

Genitourinary diseases

Cystic fibrosis and other chronic lung diseases

Metabolic disorders

Hydrocephalus

Severe obesity

Otolaryngologic diseases

Signs and symptoms $(n=668)$

Fever

Cough

Rhinitis

Diarrhea

Pharyngodynia/pharyngitis

Vomiting

Headache

Dyspnea

Hyporexia

Conjunctivitis

Fatigue

Abdominal pain

Skin rash

Smell and taste alterations

\begin{tabular}{|c|c|}
\hline $160(21.1 \%)$ & 38 (10.2\%) \\
\hline 160 (21.1\%) & $78(21 \%)$ \\
\hline 155 (20.4\%) & 87 (23.4\%) \\
\hline 284 (37.4\%) & 168 (45.3\%) \\
\hline 426 (56.1\%) & 205 (55.3\%) \\
\hline 333 (43.9\%) & 166 (44.7\%) \\
\hline 136 (17.9\%) & 50 (36.8\%) \\
\hline 20 (14.7\%) & $3(6 \%)$ \\
\hline 15 (11.0\%) & $8(16 \%)$ \\
\hline 15 (11.0\%) & $8(16 \%)$ \\
\hline $13(9.6 \%)$ & $4(8 \%)$ \\
\hline $11(8.1 \%)$ & $5(10 \%)$ \\
\hline $9(6.6 \%)$ & $2(4 \%)$ \\
\hline $9(6.6 \%)$ & $4(8 \%)$ \\
\hline $8(5.9 \%)$ & $4(8 \%)$ \\
\hline $8(5.9 \%)$ & $4(8 \%)$ \\
\hline $6(4.4 \%)$ & $3(6 \%)$ \\
\hline $6(4.4 \%)$ & $3(6 \%)$ \\
\hline $5(3.7 \%)$ & $1(2 \%)$ \\
\hline $4(2.9 \%)$ & / \\
\hline $3(2.2 \%)$ & I \\
\hline 2 (1.5\%) & / \\
\hline $2(1.5 \%)$ & $1(2 \%)$ \\
\hline
\end{tabular}

Arthromyalgy

Chest pain

Febrile seizures

Non-febrile seizures in known epilepsy
547 (81.9\%)

$254(38.0 \%)$

$139(20.8 \%)$

$107(16.0 \%)$

$86(12.9 \%)$

$67(10.0 \%)$

$67(10.0 \%)$

$62(9.3 \%)$

$60(9.0 \%)$

$56(8.4 \%)$

$55(8.2 \%)$

$52(7.8 \%)$

$38(5.7 \%)$

$27(4.0 \%)$

$23(3.4 \%)$

$16(2.4 \%)$

$11(1.6 \%)$

$3(0.5 \%)$
( $n=27$ )

$8.7,9.9$

\begin{tabular}{|c|c|}
\hline 117 (32.4\%) & $6(22.2 \%)$ \\
\hline 80 (22.2\%) & $2(7.4 \%)$ \\
\hline 63 (17.4\%) & $6(22.2 \%)$ \\
\hline $101(28.0 \%)$ & $13(48.2 \%)$ \\
\hline 207 (57.3\%) & $13(48.2 \%)$ \\
\hline $154(42.7 \%)$ & 14 (51.8\%) \\
\hline 80 (58.8\%) & $6(4.4 \%)$ \\
\hline 17 (21.3\%) & / \\
\hline 7 (8.8\%) & / \\
\hline 7 (8.8\%) & / \\
\hline $9(11.2 \%)$ & / \\
\hline $3(3.7 \%)$ & $3(50 \%)$ \\
\hline 7 (8.8\%) & / \\
\hline $5(6.2 \%)$ & / \\
\hline $4(5 \%)$ & / \\
\hline $4(5 \%)$ & / \\
\hline $3(3.7 \%)$ & / \\
\hline $3(3.7 \%)$ & / \\
\hline $4(5 \%)$ & / \\
\hline $3(3.7 \%)$ & 1 (16.7\%) \\
\hline $1(1.3 \%)$ & 2 (33.3\%) \\
\hline $2(2.5 \%)$ & / \\
\hline $1(1.3 \%)$ & / \\
\hline
\end{tabular}

(3.8-12.6)

tted for

$(n=361)$

(1) 
Among the 668 (88.0\%) symptomatic children, the pattern of presentation of COVID-19 differed by age (Figure 1). Fever (37.5-40 ${ }^{\circ} \mathrm{C}$ ) was the most common symptom (81.9\%), followed by cough and rhinitis (Table 1). Respiratory disturbances were reported in 347 patients (51.9\%), with dyspnea in 62. Gastrointestinal symptoms were observed in 174 patients $(26.0 \%)$.

Infants were significantly more likely to present with fever, rhinitis and/or diarrhea and/or hyporexia; on the contrary, headache, asthenia, cough, abdominal pain and smell and taste alterations showed an increasing frequency trend with age (Figure 1).

The median interval between symptom onset and first medical evaluation was 2 days (IQR 1;5).

\section{Co-infections}

Concomitant infecting pathogens were not systematically searched for: 244 children (32.1\%) were tested for concomitant infecting pathogens and a co-infection was found in 69 (28.3\%); of these, 8 (11.6\%) had multiple infections. Agents responsible for co-infection were viruses in 45 cases and bacteria in 32; in particular, respiratory viruses were Rhino/Enterovirus, Adenovirus, Bocavirus, Respiratory Syncytial Virus, Coronavirus other than SARS-CoV-2, Influenza A/B and Parainfluenza virus.

\section{Hospital Admission and COVID-19 Complications}

Barely a half of children (388/759) were hospitalized (Table 1). The vast majority $(361 / 388,93.0 \%)$ was admitted for COVID19-related reasons; of these, only $25(6.9 \%)$ were referred to hospital by a pediatrician or family doctor. Children admitted for other reasons with an incidental diagnosis of SARS-CoV2 infection were excluded from the analysis on risk factors for complicated disease.

Median duration of hospital stay of children admitted for COVID-19 was 6 days (IQR 4;11, mean 8.72 days). Hospital stay was longer in the first phase of the pandemic (March-April) than in the following months, though without statistical significance (mean 8.94 vs. 7.42 days, respectively, $p=0.14$ ).

Age was inversely related to hospital admission $(p<0.01)$ and linearly related to length of stay $(p=0.014)$. Hospital

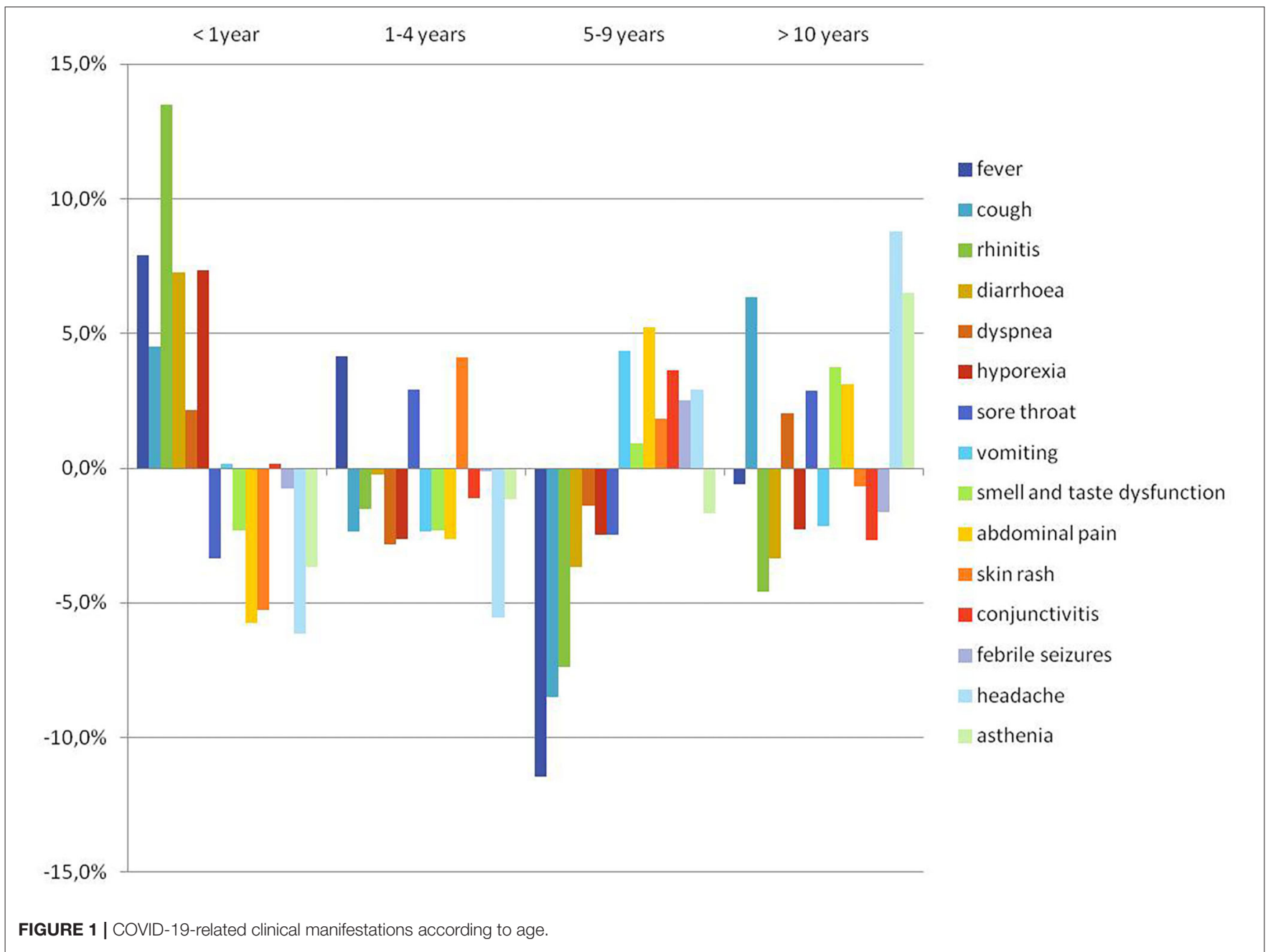


admission and length of stay were not influenced by gender ( $p$ $=0.27$ and $p=0.42$, respectively). The hospitalization rate was significantly higher among ex-preterm $(p=0.014)$ and among children with co-morbidities or immunodeficiency $(p<0.01$ and 0.009 , respectively).

One hundred and forty-nine children (19.6\% of the entire study population and $38.8 \%, 140 / 361$, of those hospitalized for COVID-related causes) developed one or more complications. Mean age was significantly higher among children with complications than in uncomplicated infections (6.9 vs. 5.5 years, respectively; $p=0.023$ ), while gender was non-contributory to complications development (Table 2). At multivariate analysis, fever or abdominal pain at onset and co-morbidities were risk factors for complications (Table 3).

Respiratory complications were reported in 123 children (16.2\%) and included pneumonia $(n=109)$, severe acute respiratory illness $(n=50)$ and acute respiratory distress syndrome $(n=5)$. Three-hundred children (39.5\%) underwent chest X-ray, with a normal finding in 148, pulmonary consolidations and/or interstitial involvement in the remaining and pleural effusions in 17 . A chest computed tomography (CT) scan was performed in 28 children $(3.7 \%)$.

TABLE 2 | Summary of the main results at univariate analysis.

\begin{tabular}{|c|c|c|c|c|c|c|c|c|c|c|c|c|}
\hline Variable & \multicolumn{3}{|c|}{$\begin{array}{l}\text { Hospital admission for } \\
\text { COVID-19 related } \\
\text { reasons }\end{array}$} & \multicolumn{3}{|c|}{$\begin{array}{l}\text { Complicated } \\
\text { SARS-CoV-2 infection }\end{array}$} & \multicolumn{3}{|c|}{ Ventilatory support } & \multicolumn{3}{|c|}{ ICU admission } \\
\hline Age (median) & 5.8 & 8.6 & $<0.001$ & 7.2 & 7.3 & 0.86 & 6.6 & 7.4 & 0.38 & 7.7 & 7.3 & 0.68 \\
\hline \multicolumn{13}{|l|}{ Age group } \\
\hline $5-9$ years & 63 & 87 & & 30 & 125 & & 13 & 142 & & 11 & 144 & 0.039 \\
\hline$>10$ years & 101 & 168 & & 52 & 232 & & 12 & 272 & & 9 & 275 & \\
\hline \multicolumn{13}{|l|}{ Demographics } \\
\hline Male gender & 207 & 205 & 0.57 & 83 & 343 & 0.9 & 26 & 400 & 0.81 & 16 & 410 & 0.73 \\
\hline Underlying clinical conditions & 80 & 50 & $<0.001$ & 47 & 89 & $<0.001$ & 23 & 113 & $<0.001$ & 14 & 122 & $<0.001$ \\
\hline Fever & 292 & 247 & $<0.001$ & 125 & 422 & $<0.001$ & 40 & 507 & 0.01 & 25 & 522 & 0.016 \\
\hline Abdominal pain & 32 & 18 & 0.026 & 24 & 28 & $<0.001$ & 7 & 45 & 0.017 & 4 & 48 & 0.095 \\
\hline \multicolumn{13}{|l|}{ Laboratory results } \\
\hline Leukocytosis & 65 & 8 & 0.024 & 44 & 34 & $<0.001$ & 18 & 60 & $<0.001$ & 11 & 67 & 0.001 \\
\hline Lymphopenia & 65 & 16 & 0.8 & 46 & 36 & $<0.001$ & 20 & 62 & $<0.001$ & 19 & 63 & $<0.001$ \\
\hline Viral coinfection & 44 & 1 & 0.016 & 21 & 24 & 0.87 & 13 & 32 & 0.015 & 8 & 37 & 0.009 \\
\hline
\end{tabular}

ICU, intensive care unit. Bold values are statistically significant.

TABLE 3 | Risk factors for hospital admission, complications, ventilatory support, and ICU admission at multivariate analysis.

\begin{tabular}{|c|c|c|c|c|c|c|c|c|c|c|c|c|}
\hline \multirow[t]{2}{*}{ Variable } & \multicolumn{3}{|c|}{ Hospital admission } & \multicolumn{3}{|c|}{ Complications } & \multicolumn{3}{|c|}{ Ventilatory support } & \multicolumn{3}{|c|}{ ICU admission } \\
\hline & $p$ & OR & $95 \% \mathrm{Cl}$ & $p$ & OR & $95 \% \mathrm{Cl}$ & $p$ & OR & $95 \% \mathrm{Cl}$ & $p$ & OR & $95 \% \mathrm{Cl}$ \\
\hline Gender & 0.714 & 0.94 & $0.69-1.30$ & 0.267 & 0.80 & $0.54-1.18$ & 0.574 & 0.83 & $0.43-1.61$ & 0.794 & 0.90 & $0.40-2.03$ \\
\hline Age & $<0.001$ & 1.11 & $1.08-1.14$ & 0.297 & 1.02 & $0.98-1.05$ & 0.087 & 1.05 & $0.99-1.12$ & 0.945 & 1.00 & $0.93-1.08$ \\
\hline Chronic comorbidities & 0.002 & 2.03 & $1.29-3.18$ & $<0.001$ & 3.01 & $1.88-4.81$ & $<0.001$ & 6.77 & $3.40-13.45$ & $<0.001$ & 4.91 & $2.11-11.42$ \\
\hline $\begin{array}{l}\text { More than one infected } \\
\text { family member }\end{array}$ & $<0.001$ & 0.48 & $0.35-0.66$ & $<0.001$ & 0.38 & $0.26-0.58$ & $<0.001$ & 0.17 & $0.07-0.42$ & 0.004 & 0.20 & $0.07-0.61$ \\
\hline Fever & $<0.001$ & 2.36 & $1.64-3.38$ & $<0.001$ & 2.58 & $1.57-4.24$ & 0.012 & 3.55 & $1.32-9.51$ & 0.026 & 5.32 & $1.23-23.10$ \\
\hline Abdominal pain & 0.001 & 3.11 & $1.62-5.96$ & $<0.001$ & 5.37 & $2.82-10.19$ & 0.012 & 3.65 & 1.33-9.99 & 0.098 & 2.75 & $0.83-9.12$ \\
\hline Immunosuppression & 0.109 & 2.76 & $0.80-9.57$ & 0.466 & 0.63 & $0.19-2.16$ & 0.998 & 0.00 & 0.00 & 0.715 & 0.67 & $0.08-5.80$ \\
\hline
\end{tabular}

OR, odds ratio; Cl, confidence interval; ICU, intensive care unit. Bold values are statistically significant. 
Neurological complications were recorded in 5 patients (4 worsening of known epilepsy and 1 development of autoimmune encephalitis), while none had SARS-CoV-2-driven encephalitis.

Gastroenterological complications occurred in 13 children: mesenteritis or appendicitis in 9 ( 2 underwent appendectomy), pancreatitis in 1 , and severe dehydration consequent to vomiting or diarrhea in 3.

Isolated cardiac involvement, including myocarditis and/or pericarditis, was documented in $7(0.9 \%)$ children.

Six $(0.8 \%)$ had a severe bacterial complication: five sepsis and one a retropharyngeal abscess.

MIS-C occurred as a complication of SARS-CoV-2 infection in 30 children $(3.9 \%$ ) with a median age of 6.6 years (IQR $3.4 ; 9.6$ ). The development of MIS-C was unrelated to gender, although children with MIS-C were mainly males $(21 / 30,70.0 \%, p=$ 0.60 ). Eight children with MIS-C had co-morbidities, including one with immune deficiency (Wiskott-Aldrich syndrome). Fever, gastrointestinal disturbances, rash and conjunctivitis were the most common findings at MIS-C onset, followed by sore throat, cough and dyspnea. Overall, 17/30 patients (56.6\%) had signs of cardiovascular involvement, with arrhythmia, left ventricle dysfunction, coronary aneurysms and pericardial effusions. Eleven patients $(36.7 \%)$ developed acute respiratory failure. Gastrointestinal manifestations, including severe abdominal pain and mesenteritis, occurred in 17 patients (56.7\%), while $5(16.7 \%)$ had transient neurological deterioration following a decline in general condition. Eleven (36.6\%) required respiratory support and two needed vasoactive medications.

\section{ICU Admission}

Thirty children (4.0\%) required intensive care support for COVID-19. ICU admission in the MIS-C group was 6 times higher than in other children: $20.0 \%(6 / 30)$ vs. $3.3 \%(24 / 729, p$ $<0.01$ ), respectively. Overall median ICU stay was 6 days (IQR $6 ; 11$, range 1-47) vs. 7 days in the MIS-C group.

Viral co-infections, underlying clinical conditions and age between 5 and 9 years were statistically related to ICU admission (Table 2). No association was found with gender. At multivariate analysis, the presence of co-morbidities was confirmed as a risk factor for the need of intensive care support (Table 3).

Three patients admitted to ICU required intubation and mechanical ventilation. Two children underwent extracorporeal membrane oxygenation: the first for severe respiratory distress in pneumonia, known epilepsy and neurodevelopmental delay, the second for severe ARDS and concomitant sickle-cell disease. Five children were treated with C-PAP and 9 with high flow nasal cannulae oxygen therapy.

The need for ventilator support was statistically related to the presence of underlying chronic diseases (Tables 2, 3).

Having more than one infected family member had a protective role in terms of complications, hospital and ICU admission and need for ventilatory support.

\section{Laboratory Results}

In children who underwent blood investigations (454/759, $59.8 \%$ ), an increase in C-reactive protein (CRP) was the most common finding (39.5\%), while other alterations were less frequently observed. Table 4 shows lab tests performed in patients admitted for COVID-19. D-dimer and troponin T were performed only in more severe cases. Children who needed ventilatory support and those with MIS-C were more likely to have a low lymphocytes count and/or a high CRP level as compared to children with mild-moderate disease or pneumonia $(p<0.005)$. Children with MIS-C also had significantly higher $\operatorname{ESR}(p<0.001)$, ferritin $(p<0.001)$ and $\operatorname{ALT}(p=0.001)$.

\section{Treatment}

Treatments intended for SARS-CoV-2 infection were prescribed in a half of patients, all hospitalized $(368 / 759,48.5 \%)$. The drugs most frequently used were macrolides $(31.0 \%)$, followed by hydroxychloroquine (15.2\%), lopinavir/ritonavir $(5.7 \%)$ and remdesivir (1.4\%). Systemic steroids were used in 28 cases $(7.6 \%)$ : 19 with COVID-19 complicated by MIS-C (67.9\%), 7 with acute respiratory failure in pneumonia, 1 with myocarditis and 1 with severe pneumococcal sepsis.

Anticoagulant therapy was given in 24 cases (6.5\%).

Low-flow oxygen-treatment was administered to 44 children (5.8\%), of these 18 (41.0\%) inside the ICU.

\section{Outcome}

All but three children recovered. Two children died: one with cardiomyopathy and a metabolic disorder, the other with severe heart disease and esophageal atresia. One child recovered with sequelae (persistent coronary aneurysms following MIS-C).

No recurrence of infection was reported so far.

\section{Prolonged Viral Detection in Respiratory Samples}

Among the 434 children for whom repeated SARS-CoV-2 PCR testing result was available, $65(15.0 \%)$ had a prolonged positivity of respiratory samples ranging from 14 to 46 days from the first positive result. Viral load could not be estimated as the $\mathrm{Ct}$ value was not available for all children. No significant association was found between persistent positivity and gender, multiple contacts within the household, disease severity, immunocompromised state, underlying chronic diseases and laboratory parameters. Younger children showed a tendency toward a more prolonged detection of viral RNA in respiratory samples, although without statistically significant difference.

\section{DISCUSSION}

Our multicenter study is, to the best of our knowledge, one of the largest cohorts on the characteristics of laboratory-confirmed SARS-CoV-2 infection in European children and points out some relevant issues on its evolution in the pediatric population.

First, given the inclusion of both pauci/asymptomatic children managed in the community setting and hospitalized children, it provides a quite realistic insight on the natural history of SARS-CoV-2 infection in the pediatric setting and a rough estimate of children requiring hospital admission or encountering complications.

In our infected children, the course of the disease was extremely variable, ranging from mild and benign forms 
TABLE 4 | Laboratory results of patients hospitalized for COVID-19: comparison of mild-moderate vs. severe forms requiring respiratory support.

\begin{tabular}{|c|c|c|c|c|}
\hline Laboratory test & $\begin{array}{c}\text { Total } \\
\text { n. abnormal/n. tested }\end{array}$ & $\begin{array}{l}\text { Mild-moderate disease } \\
\text { n. abnormal/n. tested }\end{array}$ & $\begin{array}{c}\text { Ventilatory support } \\
\text { n. abnormal/n. tested }\end{array}$ & $p$ \\
\hline $\mathrm{CRP}$ (> 10 mg/L) & $196 / 318$ & $99 / 283$ & $27 / 35$ & $<0.001$ \\
\hline Ferritin (> $150 \mathrm{ng} / \mathrm{mL}$ ) & 28/178 & $19 / 159$ & $9 / 19$ & 0.005 \\
\hline $\mathrm{ESR}(>20 \mathrm{~mm} / \mathrm{h})$ & 25/194 & $18 / 179$ & $7 / 15$ & 0.006 \\
\hline Leukocytes (higher than ULN according to age limits) & $57 / 319$ & $43 / 284$ & $14 / 35$ & 0.009 \\
\hline Lymphocytes (lower than ULN according to age limits) & $49 / 319$ & $36 / 284$ & $13 / 35$ & 0.005 \\
\hline $\operatorname{ALT}(>2 \times$ ULN) & $39 / 307$ & $32 / 272$ & $7 / 35$ & 0.103 \\
\hline
\end{tabular}

N, number; CRP, C-reactive protein; ESR, erythrocyte sedimentation rate; ALT, alanine transferase; ULN, upper limit of normal. Bold values are statistically significant.

to critical patterns. Approximately a half of children was hospitalized. The others were either entirely managed by general pediatricians/family doctors or evaluated in the emergency department and then discharged at home. The two groups (inpatients and outpatients) were similar as far as gender distribution is concerned, but varied for age distribution, comorbidities and clinical pictures.

Children admitted for COVID-19 were younger than outpatients: prematurity and age $<1$ year were independent risk factors for hospitalization, but not for COVID-19-related complications or intensive care admission. This finding suggests that a consistent number of hospital admissions were not due to disease severity but rather to a prudent approach by clinicians toward selected groups of patients (i.e., ex-preterms, neonates), beyond the propensity for their families to seek in hospital medical advice. Furthermore, it emphasizes the need for a robust territorial healthcare system in order to prevent emergency department congestion (9).

Compared to outpatients, children admitted for COVID19 had a higher percentage of underlying chronic diseases, predominantly congenital malformations and complex congenital syndromes.

Hospital stay was slightly longer during the first phase of the pandemic, probably reflecting the major uncertainties about the new disease and the initial difficulties in arranging quarantines.

Among children with symptoms, initial clinical manifestations were frequently non-specific and indistinguishable from those of other common viral infections and showed typical age-related patterns (10-12). Fever was the predominant feature, followed by cough, rhinitis and gastrointestinal disturbances. This is in contrast with what observed in other reports, where fever was less common (36-56\%) (5, 13). Among inpatients, fever, abdominal disturbances, dyspnea and febrile seizures were more frequent than in outpatients and generally represented the reason for hospital admission.

Complicated forms accounted for about $38 \%$ of hospitalized cases.

Older children, although less frequently admitted to hospital than infants, were at higher risk of complicated or severe diseases requiring prolonged hospital stay, with clinical pictures resembling those typically seen in adults.
Children with co-morbidities were more likely to develop complications and to need intensive care and ventilatory support. Therefore, underlying chronic diseases are confirmed risk factors for severe COVID-19 also in children (3, 14). Pulmonary complications such as pneumonia, respiratory distress syndrome and acute respiratory failure, were the most encountered ones, but occurred less frequently than in adults. In some patients with gastrointestinal involvement, the differential diagnosis included acute bacterial infections for the concomitant presence of mesenteritis, appendicitis or pancreatitis. Seizures and neurological complications also occurred, but the involvement of the central nervous system was limited to sporadic cases.

Complicated forms also included (7.7\%) exaggerated inflammatory responses potentially leading to shock and multiple organ failure and requiring intensive care support, identified as MIS-C $(6,8)$. Interestingly, some children developed MIS-C during the replicating phase of infection, suggesting that SARS-CoV-2 may trigger a sort of "cytokine storm" also in earlier stages. The clinical manifestations and the evolution of the disease did not differ between those with earlier onset and those with later pictures. Consistently with previous studies, a large number of our MIS-C cases had gastrointestinal symptoms, respiratory and/or cardiac impairment and shock, sometimes with initial similarities to Kawasaki Disease (12, 13, 15-20). Age between 5 and 9 years, the presence of fever or abdominal pain as well as leukocytosis or lymphopenia were risk factors for MIS-C development. In rare cases, MIS-C resulted in coronary aneurysms, suggesting that SARS-CoV-2 virus can induce an immune-mediated cardiac injury.

Co-infections may have a role in disease progression. In our study they were searched for in about one third of children, mostly hospitalized. Among viruses, Rhino/Enterovirus were the most frequent ones, while $M$. pneumoniae was the second most frequent bacteria; influenza virus was rare as the first SARS-CoV-2 epidemic in Italy coincided with the end of influenza season. In a systematic review, M. pneumoniae was the most common co-pathogen detected (58\%), followed by influenza virus (11\%) and respiratory syncytial virus (11\%) (21). Similarly to another European study, in our cohort viral coinfections were risk factors for severe disease requiring ICU admission and ventilatory support (3). This observation might have implications for the winter seasons, when the circulation 
of other respiratory pathogens, such as influenza virus, is likely to increase.

There are limited data on laboratory findings in children with COVID-19. In our study, barely $60 \%$ of tested inpatients showed increased CRP levels. Alterations in Troponin-T and NT-proBNP were specific markers of cardiac dysfunction in complicated cases, mainly MIS-C. Our data confirm that a lower lymphocytes count, as well as a high CRP levels in hospitalized patients, could be predictive of severe cases and need for ventilator support.

A chest X-ray was performed in about $40 \%$ of patients and CT scan in $<4 \%$. The low propensity of Italian pediatricians to perform radiological examinations derives from the attempt to reduce X-ray exposure in childhood. Moreover, as radiological findings in children are not specific to COVID-19, X-ray or CTscan are useful only to detect pulmonary complications. In this context, chest ultrasound may play an important role, although it was rarely performed in participating centers.

The great majority of hospitalized children with COVID-19 only required supportive therapy, including additional oxygen supplementation and fluid intake; only a bunch of patients were treated with antiviral or immunomodulatory drugs. Currently, most therapies remain experimental and their efficacy and safety are still debated. The role of these treatments should be better addressed through pediatric targeted clinical trials.

Our study confirms that SARS-CoV-2 infection is less aggressive in pediatric age than in adulthood and that case fatality rate in children and adolescents is very low $(4,22-24)$. In our series, all but three children completely recovered from the disease. Several hypotheses have been postulated to explain why children are less prone to severe COVID-19, including better immune response, lower prevalence of co-morbidities, higher mucosal colonization by other microorganisms interfering with the replication of SARS-CoV-2, lower expression of angiotensin converting enzyme- 2 receptors and cross-protection by antibodies through common viral infections $(25,26)$. The proportion of asymptomatic children included was comparable to what emerged from recent reviews; however, it presumably underestimates the true percentage of asymptomatic cases, as these are less likely to be identified, unless screened within contact-tracing or specific programs $(21,27)$. The inclusion of asymptomatic outpatients in our population probably explains the more optimistic results compared with other studies considering only inpatients $(10,28)$.

We observed a higher, though not statistically significant, prevalence of infection in males in all age groups, similarly to other pediatric studies, supporting the hypothesis that sexlinked genetic factors may influence susceptibility to SARSCoV-2 infection $(5,10,29)$. However, gender did not affect disease severity.

Concerning the source of infection, children had frequently a contact history with infected households, especially mothers. This is consistent with previous reports, but could reflect the epidemiological status of the first phase of epidemic in Italy, when school and sport activity closure had minimized contact opportunities outside home $(3,24,30)$. Interestingly, children with more than one infected household were less prone to develop complications and severe diseases. Further data are needed to better explain this finding, although a possible decreased pathogenicity of second/third line generation viruses might be supposed $(4,26)$.

The contribution of children to disease transmission is still under discussion. Recent findings suggest that those $<5$ years with mild/moderate COVID-19 have higher amounts of SARSCoV-2 RNA in their nasopharynx than older people (31). Although we did not perform quantitative assays, a relevant proportion of children had a prolonged detection of viral RNA in respiratory samples, regardless of symptoms or severity of disease. This may reflect a prolonged shedding of non-replicating viral particles, and may not affect contagiousness.

Our study has several limitations. First, the age limit at 18 years (childhood limit in Italy) makes results difficult to compare with papers with other age ranges. Secondly, enrolled children may not be fully representative of the Italian pediatric population affected, as our analysis includes a majority of hospitalized children and few cases managed at home. Finally, the limited number of selected subgroups of patients (i.e., those with cystic fibrosis or immunological defects) doesn't allow to draw conclusions in these settings.

In conclusion, complications of COVID-19 in children are related to the presence of co-morbidities. The length of hospital stay and the risk of complications increase with age: thus, the older the child, the closer the infection may resemble the adult one. Viral co-infections are additional risk factors for disease progression and high CRP levels and lymphopenia may be predictive markers of severe clinical pictures. Outside MIS-C, most children with COVID-19 require only supportive therapy.

\section{DATA AVAILABILITY STATEMENT}

The raw data supporting the conclusions of this article will be made available by the authors, without undue reservation.

\section{ETHICS STATEMENT}

The studies involving human participants were reviewed and approved by Comitato Etico Interaziendale AOU Città della Salute e della Scienza di Torino - AO Ordine Mauriziano di Torino - ASL Città di Torino. Written informed consent to participate in this study was provided by the participants' legal guardian/next of kin.

\section{AUTHOR CONTRIBUTIONS}

SG, AL, LPiera, FC, FM, AM, EC, GV, DD, SB, ID, EV, EF, RG, MD, LPierr, GN, CMo, AK, CMa, P-AT, GP, $\mathrm{ML}, \mathrm{AV}$, and GC contributed to filling-in the registry forms on patient information. SG, GP, and MD were also responsible for data entry and elaboration. All authors, including those listed in the SITIP-SIP SARSCoV-2 pediatric infection study group, contributed to the conception of the work, the acquisition of data, critical revision of the intellectual content, and also read and approved the final version. 


\section{COLLABORATORS}

Caldarelli Valeria, Salvini Filippo Maria, Corsello Giovanni, Del Barba Paolo, Berlese Paola, Agostoni Carlo, Ghizzi Chiara, Quadri Vera, Riscassi Sara, Rabbone Ivana, Calitri Carmelina, Abbagnato Luisa, Parola Luciana, Banderali Giuseppe, Pacati Ilaria, Cherubini Simonetta, Lippi Paola, Guala Andrea, Agostiniani Rino, Cazzato Salvatore, Kantar Ahmad, Baroero Luca, Rossi Nadia, Dalmazzo Cristina, Panigati Laura, Manzoni Paolo, Vigo Alessandro, Fiore Ludovica, Comberiati Pasquale, Battezzati Gianna, Verna Paola, Tornese Gianluca, Gianino Paola, Bertacca Luca, Vaccaro Angelina, Zavarise Giorgio, Marchione Luigi, Gallia Paola, Di Filippo Paola,

\section{REFERENCES}

1. Johns Hopkins Coronavirus Resource Center. (2021). Available online at: https://coronavirus.jhu.edu/ (accessed February 15, 2021).

2. Castagnoli R, Votto M, Licari A, Brambilla I, Bruno R, Perlini S, et al. Severe Acute respiratory syndrome coronavirus 2 (SARS-CoV-2) infection in children and adolescents: A systematic review. JAMA Pediatr. (2020) 174:882-9. doi: 10.1001/jamapediatrics.2020.1467

3. Götzinger F, Santiago-García B, Noguera-Julián A, Lanaspa M, Lancella L, Calò Carducci FI, et al. COVID-19 in children and adolescents in Europe: a multinational, multicentre cohort study. Lancet Child Adolesc Heal. (2020) 4:653-61. doi: 10.1016/S2352-4642(20)30177-2

4. Ludvigsson JF. Systematic review of COVID-19 in children shows milder cases and a better prognosis than adults. Acta Paediatr Int J Paediatr. (2020) 109:1088-95. doi: 10.1111/apa.15270

5. Lu X, Zhang L, Du H, Zhang J, Li YY, Qu J, et al. SARS-CoV-2 infection in children. N Engl J Med. (2020) 382:1663-5. doi: 10.1056/NEJMc2005073

6. European Centre for Disease Prevention and Control. Rapid Risk Assessment: Paediatric Inflammatory Multisystem Syndrome and SARS-CoV-2 Infection in Children. (2020). Available online at: https://www.ecdc.europa.eu/sites/ default/files/documents/covid-19-risk-assessment-paediatric-inflammatorymultisystem-syndrome-15-May-2020.pdf (accessed February 15, 2021).

7. European Commission (EC). Current Performance of COVID-19 Test Methods Devices Proposed Performance Criteria (16 Apr 2020) Brussel: EC. (2020). Available online at: https://ec.europa.eu/docsroom/documents/40805 (accessed February 15, 2021).

8. Centers for Disease Control and Prevention. Emergency Preparedness and Response: Multisystem Inflammatory Syndrome in Children (MIS-C) Associated with Coronavirus Disease 2019 (COVID-19). (2020). Available online at: https://emergency.cdc.gov/han/2020/han00432.asp (accessed February 15, 2021).

9. Vergine G, Fantini M, Marchetti F, Stella M, Valletta E, Biasucci G, et al. Home management of children with COVID-19 in the Emilia-Romagna Region, Italy. Front Pediatr. (2020) 8:575290. doi: 10.3389/fped.2020.575290

10. Swann OV, Holden KA, Turtle L, Pollock L, Fairfield CJ, Drake TM, et al. Clinical characteristics of children and young people admitted to hospital with covid-19 in United Kingdom: prospective multicentre observational cohort study. BMJ. (2020) 370:m3249. doi: 10.1136/bmj.m3249

11. Song X, Delaney M, Shah RK, Campos JM, Wessel DL, DeBiasi RL. Comparison of clinical features of COVID-19 vs. seasonal influenza A and B in US children. JAMA Netw Open. (2020) 3:e2020495. doi: 10.1001/jamanetworkopen.2020.20495

12. Jiang L, Tang K, Levin M, Irfan O, Morris SK, Wilson K, et al. COVID-19 and multisystem inflammatory syndrome in children and adolescents. Lancet Infect Dis. (2020) 20:e276-88. doi: 10.1016/S1473-3099(20)30651-4

13. Qiu H, Wu J, Hong L, Luo Y, Song Q, Chen D. Clinical and epidemiological features of 36 children with coronavirus disease 2019 (COVID-19) in Zhejiang, China: an observational cohort study. Lancet Infect Dis. (2020) 20:689-96. doi: 10.1016/S1473-3099(20)30198-5
Centenari Chiara, Iacono Alessandra, Plebani Alessandro, Ferretti Marta, Rizzi Sara, Rossin Sara, Marchisio Paola, De Fanti Alessandro, De Giacomo Costantino, Ferrante Giuliana, Barera Graziano, Roberta Pajno, Martelossi Stefano, Rocchi Alessia, Francavilla Rosa, Sogno Valin Paola, Poeta Marco, Valentini Piero, Papa Raffaele, Magistà Anna Maria, Badolato Raffaele, Saffioti Carolina, Greco Maria Cristina, Costenaro Paola, Di Pietro Maria, Esposito Susanna, Pieri Giovanni, Spartà Maria Valentina, Maffini Valentina, Cursi Laura, Nunziata Francesco, Mignone Federica, Bondone Claudia, Stera Giacomo, Marolda Agostina, Fiorica Lorenzo, Sabatini Caterina, Genovese Maria Rita, Ferrari Cristina, Galli Luisa.

14. DeBiasi RL, Song X, Delaney $\mathrm{M}$, Bell $\mathrm{M}$, Smith K, Pershad J, et al. Severe coronavirus disease-2019 in children and young adults in the Washington, DC, Metropolitan region. J Pediatr. (2020) 223:199-203.e1. doi: 10.1016/j.jpeds.2020.05.007

15. Riphagen S, Gomez X, Gonzalez-Martinez C, Wilkinson N, Theocharis P. Hyperinflammatory shock in children during COVID-19 pandemic. Lancet. (2020) 395:1607-8. doi: 10.1016/S0140-6736(20)31094-1

16. Harwood R, Allin B, Jones CE, Whittaker E, Ramnarayan P, Ramanan AV, et al. A national consensus management pathway for paediatric inflammatory multisystem syndrome temporally associated with COVID-19 (PIMS-TS): results of a national Delphi process. Lancet Child Adolesc Heal. (2020) 5:13341. doi: 10.1016/S2352-4642(20)30304-7

17. Feldstein LR, Rose EB, Horwitz SM, Collins JP, Newhams MM, Son MBF, et al. Multisystem inflammatory syndrome in U.S. Children and adolescents. N Engl J Med. (2020) 383:334-46. doi: 10.1056/NEJMoa2021680

18. WHO. Multisystem Inflammatory Syndrome in Children and Adolescents With COVID-19: Scientific Brief, 15 May 2020. Geneva: World Health Organization (2020).

19. Jonat B, Gorelik M, Boneparth A, Geneslaw AS, Zachariah P, Shah A, et al. Multisystem inflammatory syndrome in children associated with coronavirus disease 2019 in a children's hospital in New York City. Pediatr Crit Care Med. (2020). doi: 10.1097/PCC.0000000000002598

20. Consiglio CR, Cotugno N, Sardh F, Pou C, Amodio D, Rodriguez L, et al. The immunology of multisystem inflammatory syndrome in children with COVID-19. Cell. (2020) 183:968-81.e7. doi: 10.1016/j.cell.2020.09.016

21. Hoang A, Chorath K, Moreira A, Evans M, Burmeister-Morton F, Burmeister F, et al. COVID-19 in 7780 pediatric patients: a systematic review. EClinicalMedicine. (2020) 24:100433. doi: 10.1016/j.eclinm.2020.100433

22. Jutzeler CR, Bourguignon L, Weis CV, Tong B, Wong C, Rieck B, et al. Comorbidities, clinical signs and symptoms, laboratory findings, imaging features, treatment strategies, and outcomes in adult and pediatric patients with COVID-19: a systematic review and metaanalysis. Travel Med Infect Dis. (2020) 37:101825. doi: 10.1016/j.tmaid. 2020.101825

23. Sisk B, Cull W, Harris JM, Rothenburger A, Olson L. National trends of cases of COVID-19 in children based on US state health department data. Pediatrics. (2020) 2020:e2020027425. doi: 10.1542/peds.2020-027425

24. Epicentro. Istituto Superiore di Sanità. L'Epidemiologia per la sanità pubblica. (2021). Available online at: https://www.epicentro.iss.it/coronavirus/sars-cov2-sorveglianza-dati (accessed February 15, 2021).

25. Felsenstein S, Hedrich CM. SARS-CoV-2 infections in children and young people. Clin Immunol. (2020) 220:108588. doi: 10.1016/j.clim.2020. 108588

26. Naserghandi A, Saffarpour R, Allameh SF. Exploring the causes of mild COVID-19 involvement in pediatric patients. New Microbes New Infect. (2020) 37:100741. doi: 10.1016/j.nmni.2020.100741

27. Liguoro I, Pilotto C, Bonanni M, Ferrari ME, Pusiol A, Nocerino A, et al. SARS-COV-2 infection in children and newborns: a systematic review. Eur J Pediatr. (2020) 179:1029-46. doi: 10.1007/s00431-020-03684-7 
28. Kim L, Whitaker M, O'Halloran A, Kambhampati A, Chai SJ, Reingold A, et al. Hospitalization rates and characteristics of children aged $<18$ years hospitalized with laboratory-confirmed COVID19 - COVID-NET, 14 States, March 1-July 25, 2020. MMWR Morb Mortal Wkly Rep. (2020) 69:1081-8. doi: 10.15585/mmwr. mm6932e3

29. Chao JY, Derespina KR, Herold BC, Goldman DL, Aldrich M, Weingarten J, et al. Clinical characteristics and outcomes of hospitalized and critically ill children and adolescents with coronavirus disease 2019 at a tertiary care medical center in New York City. J Pediatr. (2020) 223:149.e2. doi: 10.1016/j.jpeds.2020.05.006

30. Garazzino S, Montagnani C, Donà D, Meini A, Felici E, Vergine $\mathrm{G}$, et al. Multicentre Italian study of SARS-CoV-2 infection in children and adolescents, preliminary data as at 10 April 2020. Euro Surveill. (2020) 25:2000600. doi: 10.2807/1560-7917.ES.2020.25.18. 2000600

31. Heald-Sargent T, Muller WJ, Zheng X, Rippe J, Patel AB, Kociolek LK. Age-related differences in nasopharyngeal severe acute respiratory syndrome coronavirus 2 (SARS-CoV-2) levels in patients with mild to moderate coronavirus disease 2019 (COVID-19). JAMA Pediatr. (2020) 174:9023. doi: 10.1001/jamapediatrics.2020.3651

Conflict of Interest: The authors declare that the research was conducted in the absence of any commercial or financial relationships that could be construed as a potential conflict of interest.

Copyright (c) 2021 Garazzino, Lo Vecchio, Pierantoni, Calò Carducci, Marchetti, Meini, Castagnola, Vergine, Donà, Bosis, Dodi, Venturini, Felici, Giacchero, Denina, Pierri, Nicolini, Montagnani, Krzysztofiak, Bianchini, Marabotto, Tovo, Pruccoli, Lanari, Villani, Castelli Gattinara and the Italian SITIP-SIP Pediatric Infection Study Group. This is an open-access article distributed under the terms of the Creative Commons Attribution License (CC BY). The use, distribution or reproduction in other forums is permitted, provided the original author(s) and the copyright owner(s) are credited and that the original publication in this journal is cited, in accordance with accepted academic practice. No use, distribution or reproduction is permitted which does not comply with these terms. 